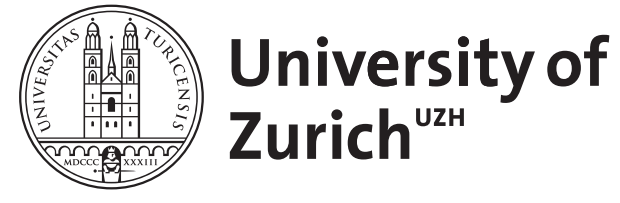

\title{
Wüste. Kleiner Rundgang durch einen Topos der Askese
}

Keller, Hildegard Elisabeth

DOI: https://doi.org/10.1515/9783110228373.4.191

Posted at the Zurich Open Repository and Archive, University of Zurich

ZORA URL: https://doi.org/10.5167/uzh-46128

Book Section

Published Version

Originally published at:

Keller, Hildegard Elisabeth (2010). Wüste. Kleiner Rundgang durch einen Topos der Askese. In: Röcke, Werner; Weitbrecht, Julia. Askese und Identität in Spätantike, Mittelalter und früher Neuzeit. Berlin, Germany: de Gruyter, 191-207.

DOI: https://doi.org/10.1515/9783110228373.4.191 


\title{
Wüste. Kleiner Rundgang durch einen Topos der Askese
}

\author{
HiLDEGARD ELISABETH KELLER
}

Die großen Sand-, Fels- und Steinwüsten dieser Erde verkörpern - im Verbund mit den sogenannten Eiswüsten der Polkappen - den radikalen Gegenpol zu den besiedelten und agrikulturell genutzten Zonen der Erde. Ihr Charakteristikum ist eine extreme physikalische, biologische und soziale Reduktion. Der Ausdruck >Wüste< wird zu einem Synonym von Leere, Nichts. Das mittelhochdeutsche Wort bzw. seine morphologischen Varianten bildet die Grundlage für ein faszinierendes Kapitel innerhalb der mittelalterlichen Begriffsgeschichte der Natur. Es bezeichnet den geophysikalischen Ort, die rhetorische Figur der expressiven Allegorie >Wüste und Denkmodell der negativen Theologie. Der vorliegende Beitrag stellt somit Wüsten-Topoi im buchstäblichen und im übertragenen Sinn vor.

Die begriffsgeschichtlichen Ausführungen zu >Wüste $<$ beziehen sich einerseits auf die genuine, topographische Bezeichnung für aride Landstriche, hier insbesondere die Sand- und Steinwüsten des Mittelmeerraums und des Nahen Ostens - dorthin sollen sich die ersten Eremiten in der christlichen Spätantike zurückgezogen haben -, andererseits auf die in der Wüste entstandene Literatur. Kaum zufällig finden sich im Kontext der spätmittelalterlichen Rezipienten der Wüstenväter-Literatur auch Texte, welche die Sprache selbst einer asketischen Übung unterziehen. Der Ausdruck >Wüste< spielt hierin die zentrale Rolle, denn er dient darin als Metapher für alles der Bezeichenbarkeit Entzogene. Dies signalisiert ganz grundsätzlich die Möglichkeit, dass ein semantisch definiertes lexikalisches Element auf seinen Bezeichnungsanspruch verzichtet - eine Art Askese, welche Folgen für die Sprache selbst haben müsste.

Der Beitrag geht zunächst auf die begriffsgeschichtliche Verbindung zwischen der topographischen Bezeichnung > Wüste $<$ und den ersten Ansätzen christlicher Askesepraxis in der Spätantike ein. Dazu gehört auch die kritische Diskussion der immer wieder behaupteten Genese des asketischen Lebensmodells aus dem Leben in der Wüste. Im Anschluss daran wird auf die literarische Produktivität der Wüste eingegangen, der eine lang anhaltende, im Spätmittelalter besonders wichtige Rezeption folgte. Gleichzeitig werden Forschungspositionen erwähnt, welche die Behauptung, zwischen der Wüste und der Genese christlicher Askese bestehe ein ursächlicher Zusammenhang, dementieren. 


\section{Geographische und imaginäre Topographie}

Sand-, Stein- und Felswüsten machen rund ein Fünftel der festen Erdoberfläche aus. Sie stellen allerhöchste Ansprüche an die Durchreisenden. Sie werden auch zu einem Topos der Unwegsamkeit. Deshalb wurden im Mittelalter zwei Naturräume in Verbindung gebracht, die auf den ersten Blick nicht unähnlicher sein könnten: die (leere) Wüste und der (dichte) Wald. ${ }^{1}$ Die Wüste wird als erschreckender Ort des Entzugs von allem vital Notwendigen erlebt und verweist deshalb auch auf intellektuelle und seelische Ödniserfahrungen: Einsamkeit, Langeweile, Isolation, Stumpfheit und Unansprechbarkeit sowie jede nicht näher bestimmbare Erfahrung des Nicht-Erfahrens. Wer in die Wüste geschickt wird, erleidet eine Strafe, die existenzbedrohend sein kann. Interessanterweise zeigt die Literatur immer wieder, dass die Übergänge zwischen der Wüste als realem bzw. imaginärem Ort fließend sind.

Ödland und Wüsten spielen im Alten Testament eine wichtige Rolle. Eine eigentliche Wüstentheologie entsteht im Kontext der vierzig Jahre dauernden Wanderschaft durch die Wüste, die über das Volk Israel verhängt worden war. ${ }^{2}$ Der Wüstenbegriff ist vieldeutig und kann einen geografischen Ort, eine Zeitspanne und eine innerlich oder gesellschaftlich bedingte Situation der sich dort Aufhaltenden bedeuten, wie im resümierenden fünften Buch Mose bzw. Deuteronomium deutlich wird. Vierzig Jahre in der Wüste zu leben, bedeutete für das Volk Israel eine generationelle Zäsur, da sich die Zahl der Handlungsfähigen in dieser Zeitspanne biologisch erneuert hatte. Es war eine Gefahr für die Identität, dass jene, die das kollektive Geschick bestimmt und somit auch die kollektive memoria getragen hatten, nicht mehr waren. Zu den physischen Strapazen und zur Gefahr der sozialen Isolation drohte nun auch das Vergessen der eigenen Geschichte. Diese kulturelle Auslöschungsgefahr zählte wesentlich zur Schrecklichkeit der Wüste und konstituierte sie als einen Raum der Strafe, des Wartens und der Erneuerung. Dieser existentielle Entzug schlechthin charakterisiert die negativ geprägte Wüstentheologie des Alten Testaments. Für das hier Diskutierte muss jedoch im Auge behalten werden: Der Gang durch die Wüste stellt eine Vorbereitung auf das Land Kanaan dar - Fülle bzw. Erfüllung und Entzug bzw. Leere sind untrennbar verbunden. Diese Koppelung prägt auch die Wüsten-Semantik in den mystischen Texten.

Auch im christlichen Kulturraum ist der Wüstenbegriff ambivalent und verbindet Verworfenheit mit Heilserwartung und Erlösung. Dies wird sich bei den frühchristlichen Anachoreten und Eremiten in den Sand-, Stein- und Felswüsten des Mittelmeerraums ebenso zeigen wie bei den hoch- und spätmittelalterlichen Eremiten und Einsiedlern im funktional adäquaten Habitat, dem Wald.

1 In den mittelhochdeutschen Bezeichnungen für $>$ Wüste`, die durch das ganze Mittelalter hindurch synonym für $>$ Wald verwendet werden konnten, wird ihre wortgeschichtliche und kulturhistorische Verwandtschaft evident. Vgl. Keller (2008a) und (2008b).

2 Gomes de Araujo (1999), 23-33. 
Noch in späteren Jahrhunderten übernahmen andere aus der Gesellschaft Ausgeschlossene die religiös fundierte Identität als Wüstenbewohner. Die sogenannten déserts der Hugenotten bezeichneten der Historiografie des französischen Protestantismus folgend sowohl einen Zeitraum der Verfolgung (zwischen der Revokation des Edikts von Nantes 1685 bis zum Beginn des 18. Jahrhunderts) als auch die siedlungsfernen Zufluchtsorte, in welche die verfolgten Hugenotten flohen (Cevennen, Vivarais, Dauphiné). Ihre Selbstdefinition als Widerstandskämpfer in der Wüsteく rekurriert auf das Geschick des auserwählten Volkes Israel unter Moses. Sie identifizierten sich mit dessen vierzig Wanderschaftsjahren in der Wüste und deuteten ihre eigene Situation als analoge Prüfung und Gemeinschaft mit Gott, ebenfalls als »a period of transition «, die in ihrer >rite de passage<-Funktion selbst eine genuine Gotteserfahrung darstellte: »Although divine judgement was a tragic part of that relationship, the fact remains that the wilderness period was a time when leadership and instruction came from God, through the divinely appointed leaders. $\ll^{3}$ Das Frankreich des ausgehenden 17. Jahrhunderts wurde somit in einer schwer auflösbaren Verquickung von geografischer Referenz, theologischer und konfessionspolitischer Metapher zu >la nouvelle Égypte ${ }^{4}{ }^{4}$

Doch Wüsten, unzugängliche Waldgebiete und verödete Landstriche ermöglichten immer auch den freiwilligen Gang in die Wüste.

\section{Wege in die Wüste}

Von der Spätantike bis in die Moderne verließen Menschen aus freien Stücken ihre Heimat, ihren angestammten Lebensraum des Dorfes oder der Stadt, um sich in unbesiedelte Gebiete zurückzuziehen. Ein solcher, radikaler Schritt erfolgte kaum je grundlos. In den hier betrachteten Fällen sollte er in aller Regel im religiösen Sinn identitätsstiftend sein bzw. den für die Identität der jeweiligen Gruppe adäquaten Verwirklichungsraum schaffen. Dies lässt sich in der an die Waldwildnis geknüpften Gründungsgeschichte des Zisterzienserordens ebenso nachweisen wie in der Popularität des spätmittelalterlichen Eremitentums. Noch die Biografie des modernen Wüstenvaters Charles de Foucauld (1858-1916) ist davon geprägt: Er zog sich nach einer Militär- und dann einer Trappistenlaufbahn als Eremit in die algerische Sahara (Tamanrasset) zurück. Dazu soll ihn seine Rückbesinnung auf die frühen Wüstenmönche und auf seine militärischen Erlebnisse bewogen haben: »I love this desert; it is so good and wholesome to be alone, face to face with eternal things - truth washes over you like a flood. $\ll^{5}$

3 Burden (1994), 231.

4 Vgl. Bost (2000) und Keller (2008a).

5 de Foucauld, Notat vom 15. Juli, 1906, zitiert nach de Foucauld (1999), 116. 
Er fand am 1. Dezember 1916 den gewaltsamen Tod durch eine Tuareg-Rebellengruppe; seine Schriften wurden postum ediert, und auch die Ordensgründung der >Pères du désert $\iota\left(\right.$ Wüsten-Patres $\measuredangle$ ) erfolgte erst nach seinem Tod. ${ }^{6}$

Unbestrittenermaßen spielte die Antithese zwischen den Siedlungszentren und peripheren, unbewohnten Landstrichen eine wesentliche Rolle für die Selbstdefinition des Christentums innerhalb der spätrömischen Staatsstrukturen und der Religionen im Mittelmeerraum, mit denen es konkurrierte. ${ }^{7}$ Wüstenlandschaften wurden als »religionsfreundlich, wenn nicht gar religionsproduktiv « ${ }^{8}$ bezeichnet. Der alttestamentliche Gott machte die Wüste zum Straf- und Prüfungsort für das Volk Israel, er strafte aber auch dessen Feinde mit gezielter Verwüstung von Kulturland. So muss der Prophet Ezechiel dem Pharao ankündigen, das fruchtbare Land am Nil werde für 40 Jahre zu Ödland, und er selbst werde wie ein Seeungetüm, an dessen Schuppen sich die anderen Fische verbissen haben, herausgezogen und aufs Trockene gesetzt (Ez 29,1-12). Das Neue Testament erhob die Wüste zum Ursprungsort messianischer Bewegung. Dies lässt sich nicht nur im Blick auf Johannes den Täufer sagen (vgl. Mk 1, 3; Mt 24,26), sondern auch in Bezug auf die Urszene von Christi dreifacher Versuchung in der Wüste (Mt 4, 3-9; Lk 4, 3-12).

In den ersten frühchristlichen Jahrhunderten zogen sich Christen in nordafrikanische, syrische und ägyptische Wüstenstriche zurück und begründeten jene Frühformen asketischen Lebens. Sie wurden zu institutionellen Vorformen der mittelalterlichen Einsiedler- und Klosterkultur, aus der die erste und für lange Zeit einzige intellektuelle und spirituelle Elite des christlichen Europa hervorgehen sollte. ${ }^{9}$ In Ägypten, dann auch auf der Halbinsel Sinai, in Palästina, Syrien und schließlich in Kleinasien bot sich eine >Welt außerhalb der Welt.${ }^{10}$ Der von den frühchristlichen Asketen gewählte Lebensraum war ein Gegenpol zu den städtischen Zentren der Zivilisation. Für die Bewohner der Städte und Dörfer muss die Flucht in die unbewohnten Gegenden provokant gewesen sein, denn sie verkehrte die gewohnten Sichtweisen auf die soziale Ordnung: Erstens lehnten die Wüstenbewohner die Lebensumstände mit allen zivilisatorischen Errungenschaften in den Siedlungszonen ab; zweitens erschien der von allen Kulturanstrengungen ausgesparte, von der Gesellschaft geächtete Raum nun plötzlich als heilsträchtiger Ort. Religionssoziologen deuteten die Entscheidung der Wüstenbewohner deshalb als »Selbststigmatisierung « ${ }^{11}$ Der Begriff bezeichnet ihre freiwillige soziale Devianz. Die Wüstenbewohner nahmen soziokulturelle Stigmata in Kauf; wie im

$6 \quad$ Vgl. de Foucauld (1999).

7 Stolz (2001), 166 f.; vgl. Brown (1994).

8 Donner (1997).

9 Vgl. Fragonard (2000), 163; Brunert (2000).

10 Rahner (1999), Teil C »Aszese und Mystik in der Väterzeit«.

$11 \mathrm{Zu}$ dem von Wolfgang Lipp geprägten Begriff der Selbststigmatisierung und zu seiner Relationierung mit dem Begriff `Charisma s. Lipp (1985), 7-36; vgl. a. Lipp (1975), 25-53; Mödritzer (1994). 
Falle des in einem härenen Kleid auftretenden Johannes des Täufers waren es minderwertige oder auch gegen die Norm verstoßende Kleider, der Aufenthalt in Lebensräumen, die andere mieden, sowie soziale Ächtung. Die Geste drückte den religiös motivierten Widerstand gegen das nach materiellem Besitz und Macht rangierende spätrömische Sozialsystem aus. ${ }^{12} \mathrm{Zu}$ den Paradoxien des Wüstenlebens gehört die Öffentlichkeitswirkung: Die Wüstenasketen zogen oft schon nach kurzer, in der Einöde verbrachter Zeit Menschen an, die in ihnen charismatische Lehrer und Propheten sahen. Von diesem Kausalkonnex zwischen Stigmata und Charisma zehrt auch die literarische Produktivität des Wüstenlebens. Davon wird noch zu sprechen sein.

Im Blick auf das soeben Dargestellte verwundert es nicht, dass die Entstehung der frühchristlichen Askesemodelle in einen Kausalzusammenhang mit der Wüste gebracht wurde. Der amerikanische Religionswissenschafter James Goehring übte in mehreren Publikationen Kritik daran. ${ }^{13}$ Das im späten 3. und 4. Jahrhundert dokumentierte frühchristliche Mönchtum in Ägypten sei kein primär an die Wüste gebundenes Phänomen. Mit Belegen aus der griechischen Literatur der Spätantike suchte Goehring nachzuweisen, dass ein bereits viel früher geprägter Topos die Verworfenheit des Leben in der Stadt der wertpositiven Einfachheit des Wüstenlebens gegenübergestellt hat (in vergleichbarer Weise wie bukolische Motive dazu dienten, das Leben am Hof bzw. in den Städten zu desavouieren). Eine Handlungsanweisung für Zivilisationsflüchtlinge, die durch den Gang in die Wüste zu den ersten christlichen Asketen geworden wären, wollte Goehring darin nicht erkennen. Gleichwohl machte er darauf aufmerksam, dass die etablierte Topik der Wüste in den ersten frühchristlichen Jahrhunderten zur Lebenswirklichkeit wurde, und zwar in den Biografien der ersten Wüstenasketen: »In fourthcentury Egypt, the metaphor was translated into radical reality as ascetic Christians embraced a life which demanded spatial withdrawal (gr. anachóresis) from the social world of the villages and towns to the isolation of deserts. ${ }^{14}$ Asketische Lebensmodelle aber seien bereits vor dem Auszug der Anachoreten und Koinobiten in die Wüste in urbanen und periurbanen Räumen fassbar.

Goehring sieht in den Begriffen >Wüste < bzw. >Stadt « keine Dichotomie, sondern die beiden Extrempole eines Kontinuums. Sein literarischer Kronzeuge hierfür ist die Vita des ersten namentlich bekannten Wüstenmönchs. Der spirituelle Vervollkommnungsweg des koptischen Eremiten Antonius (gest. 356) und sein physischer Rückzug in immer verlassenere Landstriche, schließlich bis tief in die Wüste hinein werden als parallele Bewegungen gezeigt. Das sukzessive Eindringen sowohl in das eigene Innere als auch in die Wüstentopographie entspricht einem Prozess, der die Antonius-Vita mit mystischen Texten aus dem Spätmittelalter verbindet: Der Weg in die Gottheit wird als ein Itinerar durch die Wüste visualisiert. Diese Topik des Lebensraums Wüste, die nunmehr alle wertpositiven

12 Zu den sichtbaren Zeichen vgl. das bei Mödritzer (1994), 49-56 zusammengetragene Material.

13 Vgl. Goehring (1993), (1999) und (2003).

14 Goehring (1999), 73. 
wie -negativen Aspekte von Buße bis Perfektion umfasste, konnte in der monastischen Anthropologie zum klassischen Topos für die Unermesslichkeiten des homo interior, des inneren Menschen, werden. ${ }^{15}$

\section{Erzählungen aus der Wüste}

Es ist den Erzählungen, Spruchsammlungen und Geschichten aus der Wüste zu verdanken, dass das christliche Askesemodell und die Wüste verbunden sind. Jene Asketen, die sich in die Wüste zurückgezogen haben, produzierten Texte, die populär wurden und weite Verbreitung fanden. Dies steht im Gegensatz zu den urbanen bzw. periurbanen Asketen. Bereits um Antonius, den frühesten unter den bekannten Wüstenbewohnern, rankte sich die nicht allein berühmteste Lebenserzählung - die Antoniusvita regte zahllose weitere Schreiber sowie auch Illuminatoren und Maler an.

Bereits die ersten Berichte über die Wüstenasketen machten die Wüste zum Ort des zähen, ja unerbittlichen Ringens um Vervollkommnung. Diese Topik des heilsträchtigen Lebens regte ihrerseits zu einer immensen Literaturproduktion an. Sie umfasste vorbildhafte Einzelviten, Schilderungen eremitischen Lebens, Spruchsammlungen der Väter und Historiografien. Die Texte entstanden zunächst auf Griechisch, wurden später ins Lateinische und in verschiedene Volkssprachen übersetzt. Ungeachtet von Goehrings Kritik darf die Wüste somit im Blick auf die in ihr lebenden Asketen sowie deren Verehrer als ein literarisch produktiver Ort bezeichnet werden. ${ }^{16}$ Die Texte ließen den Rückzug in die Wüste (später auch den Einschluss hinter Klostermauern) zu einem Synonym für asketische Perfektionsstrategien werden: »The desert monk became the icon of renunciation in Egypt. ${ }^{17}$

Das Leben des Antonius wurde bald nach dem Tod durch Athanasius (gest. 373) niedergeschrieben. Zwar gilt er nicht ganz zu Recht als der ägyptische Mönchsvater, ${ }^{18}$ doch ist zweifellos richtig, dass diese Biografie den Beginn der asketischen Wüstenliteratur markiert: »[...] the Life of Antony really is the story of the Word in the desert. « ${ }^{19}$ Diese Antoniusvita erzählt vom Phänomen der Gren-

15 Auch hier gibt es Verbindungen einerseits zum Begriff des Waldes in der geistlichen Literatur des Mittelalters (s. Keller [2008b]), andererseits zur theologischen Metapher des Meeres (s. McGinn [1994]).

16 Die aus der Wüste überlieferten Viten- und Spruchsammlungen kursierten im Mittelalter unter verschiedenen griechischen, lateinischen und deutschen Bezeichnungen (Vitaspatrum bzw. Vitae patrum und altvetter buoch; Verba seniorum bzw. Apophthegmata patrum; Historia monachorum). Vgl. hierzu Williams/Hoffmann (1999).

17 Goehring (1999), 87-88.

18 Brunert (2000). Zur Vita siehe Bartelink (1994), deutsche Übersetzung in: Gottfried (1987).

19 Vivian (1995), 1. 
ze und des Jenseits-Davon ${ }^{20}$ und wird für die Mönche des Westens und ihre hagiographische Viten- und Legendenliteratur exemplarisch. Gemäß Athanasius' nicht ganz wahrheitsgetreuer Stilisierung zog Antonius sich als erster Mönch ins Sandgebirge am rechten Nilufer zurück, und zwar im Anschluss an intensive Bibelmeditation. ${ }^{21}$ Sein Lebensbericht zeigte den polaren Gegensatz zwischen einer sozial bestimmten Lebensweise und einer jeder personalen und materiellen Bindung entsagenden Nachfolge Christi. Antonius wählte die Wüste, um die radikale Freiheit gerade auch unter erschwerten äußeren Bedingungen zu erringen. Hilfe und Heil erwuchsen ihm auf geistigem Weg, sei es bei Wassermangel, den er im Gebet wundersam behob (vgl. Kap. 54) oder bei den berühmt gewordenen Attacken durch Dämonen (Kap. 51-53). Vom Flussufer her entwickelte sich schon bald ein reger Besucherstrom, der den dämonologischen Belehrungen des innerlich wie äußerlich geprüften Eremiten lauschte. Die Wüste erschien in Antonius' Vita als ein Ort des permanenten Kampfes und zugleich des Immer-imAnfang-begriffen-Seins. Die in der Wüste praktizierte theoria der eigenen Affekte und Leidenschaften mündete in eine Vielzahl von didaktischen Forschungsberichten über die Wahrnehmung des inneren Menschen, seine sichtbaren und unsichtbaren Feinde und über die möglichst günstigen Bedingungen zur Selbst- und Gottes-Erkenntnis. ${ }^{22}$

Benedikt von Nursia (um 480-547) verfasste, nachdem er mehrere Jahre als Eremit in einer schwer zugänglichen Höhle im italienischen Aniotal verbracht hatte, jene Regula, die zum Gründungstext des institutionalisierten Klosterlebens des Westens werden sollte. Das erste Kapitel unternahm eine Bestandsaufnahme der Benedikt bekannten mönchischen Lebensformen. Er systematisierte vier Typen eines asketischen Lebensmodells. Seine Rangfolge lässt erkennen, dass er allein die erst genannte Form (die Koinobiten, also die gemeinschaftlich unter Regel und Abt Dienenden) uneingeschränkt gelten ließ. Die letzten beiden verachtete er unverhohlen (die Sarabaiten, die er als eine Art willkürliche Heuchler sieht) und die Gyrovagen (herumstreunende Mönche, die sich in andern Klöstern aushalten lassen). ${ }^{23}$ Hochachtung zollte er auch den eremitisch lebenden Anachoreten. Diese lebten in der Wüste, zwar »nicht [mehr] in der ersten Begeisterung für das [gemeinschaftliche] Mönchsleben«, aber als monastische Experten waren sie darin geübt, »gegen den Teufel zu kämpfen«. Die Regula erkannte sie als gemeinschaftserfahrene Einsiedler an, die nach Jahren klösterlichen Lebens sich in die Einsamkeit der Wüste zurückgezogen hatten:

20 Tim Vivian spricht in seiner Einleitung von »frontier work«, Rowan Williams im Vorwort von "a narrative that positions itself on the cutting edge of a new movement, pushing back existing boundaries « (Vivian [1995], i).

21 Mt 19, 21-26.

22 Vgl. Evagrios Pontikos, Briefe, 135-139.

23 Benediktsregel, Kap. 1. 
In der Reihe der Brüder wurden sie gut vorbereitet für den Einzelkampf in der Wüste. Ohne den Beistand eines anderen können sie jetzt zuversichtlich mit eigener Hand und eigenem Arm gegen die Sünden des Fleisches und der Gedanken kämpfen, weil Gott ihnen hilft. ${ }^{24}$

Die beiden von Benedikt favorisierten spirituellen Lebensformen, deren Kontinuität bis in die Gegenwart hineinreicht, haben im mittelhochdeutschen Sprachgebiet des Hoch- und Spätmittelalters Texte hervorgebracht, die von realer Wüsten- und Wildniserfahrung eindrücklich zeugen und direkt oder indirekt von der spätantiken Wüstenberichten inspiriert sind. ${ }^{25}$ Sie erfuhren eine intensive Rezeption und zogen Imitatoren an, die ihrerseits wiederum zu verehrungswürdigen Vorbildern für die Zeitgenossen wurden. Die Wüstenmönche dienten im monastischen Kontext des Spätmittelalters als Figuren des asketischen und spirituellen Kampfes: Wer sich physisch in die Wüste bzw. innerhalb der Klostergemeinschaft in die Einsamkeit zurückzog, erfuhr die ungehindert über ihn hereinbrechenden inneren Dämonen. Auch hierfür war Antonius das durch das ganze Mittelalter präsente Ur- und Vorbild in einem.

Im deutschen und lateinischen Werk von Heinrich Seuse (vermutlich 12951366) lässt sich die literarische Funktion der frühchristlichen Wüstenväter besonders gut dokumentieren. Die Spiritualität der sogenannten altvetter (Alt- bzw. Wüstenväter) ist ein Kernelement in Seuses mystischer Lehre. Als Schüler von Meister Eckhart (1260-1328) muss ihm der spekulative, von der negativen Theologie geprägte Wüsten-Begriff seines Lehrers Meister Eckhart geläufig gewesen sein, ${ }^{26}$ doch soll an dieser Stelle die Traditionslinie zu den Wüstenvätern weiterverfolgt werden. Im Horologium sapientiae zollt Seuse dem Altvater Arsenius hohes Lob - er sieht in ihm den summus philosophus, denn dessen Lehre enthalte den nucleus totius perfectionis. ${ }^{27}$ Am herausragendsten aber ist die Präsenz der Wüstenasketen in Seuses Vita, denn sie überformt maßgeblich den spirituellen Werdegang des Protagonisten, namentlich dessen erste Lebenshälfte: Sie stand im Zeichen einer extremen Askesepraxis, die den Körper massiv in Mitleidenschaft zog. ${ }^{28}$

Der Protagonist der Vita - ein anonymer Dominikaner, der »Diener der ewiger Weisheit« genannt wird - entdeckte als junger Mönch dank der Lektüre asketischer Texte, unter denen sich auch die Wüstenväter-Viten befanden, die Zurückgezogenheit innerhalb der Klostergemeinschaft und entwickelte ausgeklügelte

24 Benediktsregel, Kap. 1, 3-5.

25 Zur Rezeption der Vitaspatrum s. Kunze/Williams/Kaiser (1987) und Petroff (1994). Zu den Altvätersprüchen s. Miller in: Weisung der Väter (2002); Müller (2000).

26 Zu Eckharts Wüsten-Semantik s. McGinn (1994); Dietrich (1994), 31-43; Asmuth (2000); Lindemann (2000), 90-96.

27 Vgl. Gnädinger (1998).

28 Die Vita ist der erste, im Exemplar enthaltene Text (Edition in Seuse [1961]). Eine Audiofassung dieses stilisierten Lebensberichts ist enthalten in Keller (2007); im Begleitbuch sind die Exemplar-Handschriften und ihr Bildprogramm besprochen, die Illuminationen sind in Farbe reproduziert. 
Übungen des Schweigens, des Nahrungs- und Schlafentzugs sowie körperliche Peinigungen, die im Laufe der Jahre ans Lebendige gingen. Der Protagonist definierte das vom Bodensee umspülte Konstanzer Inselkloster zur Wüste um, ${ }^{29}$ beschäftigte sich intensiv mit den Texten der Wüstenväter (die in die Vita integrierten Sprüche sind in den Exemplar-Handschriften durch Rubrizierung hervorgehoben) und ließ Wandgemälde und Sprüche von Wüstenvätern an die Kapellenwände malen; ein Wunder bestätigte die Autorität dieser Vorbilder. Später, als er selbst längst zum spirituellen Lehrer für seine exemplarische Schülerin, die Dominikanerin Elsbeth Stagel, geworden war, sandte er ihr vierzig Altvätersprüche ins Kloster Töss. Sie befand sich auf einem Vervollkommnungsweg, der demjenigen des Dieners nachgebildet war; nach Auffassung ihres Lehrers sollte sie jedoch auf die harte Askesepraxis zugunsten verinnerlichter Tugendübungen verzichten.

Seuse nutzte auch die Wüste als expressive Allegorie, um seine Schülerinnen aus Laienkreisen und Klöstern von der Notwendigkeit der spirituellen Sorge um sich selbst zu überzeugen. Er sprach die Adressatinnen an als »zarte wingarten des himelschen vatters«, die allzu lange wuiste gelegen und nun voller Dorngestrüpp und Ungeziefer seien. ${ }^{30}$ Die Semantik des alemannischen Adjektivs wüste besitzt eine ästhetische Komponente (`häßlich $\iota)$ und spielt indirekt auf die makellose Schönheit der Hoheliedbraut an. Dieselbe ästhetische Konnotation schwingt mit, als der Diener in der Vita seine Schülerin Elsbeth anleitet, von der wuisti eins vihlichen unbekanten lebens hin zum geheissen land eins lutren rüwigen herzen ${ }^{31}$ fortzuschreiten - eine imaginäre Wanderung durch die Wüste, in den Fußstapfen des Volkes Israel.

Nach Seuses Tod kommt dessen Charisma als spirituelle Autorität, von der im Kontext der Wüstenväter-Literatur bereits die Rede war, zum Tragen. Die Rezeption von Seuses Schriften ist nach Ausweis sowohl der handschriftlichen als auch der gedruckten Überlieferung überaus erfolgreich und richtet sich an Menschen, die Orientierung in einer asketischen Praxis suchen. Noch in der Druckgeschichte des Exemplars finden sich Empfehlungen an die Leser, unter denen die Eremiten und Brüder in den Einöden explizit angesprochen sind. ${ }^{32}$

Die Wirkungsgeschichte der Wüstenväter-Literatur reicht über die großen Schwellen der frühen Neuzeit - die Erfindung des Buchdrucks und die Reformation - hinaus. Die Wüstenväter-Texte wurden im 15. und 16. Jahrhundert oft gedruckt; Martin Luther soll eine Neuausgabe einer lateinischen Sammlung veranlasst haben. Die Auswahlbearbeitung schließlich, die der Pietist Gottfried Arnold im Jahre 1700 herausgab, wurde noch im 18. Jahrhundert rege gelesen, unter an-

29 Hamburger (2007).

30 6. Brief des Großen Briefbuches, in: Seuse (1961), 425.

31 Vita, Kap. 46, in: Seuse (1961), 156, 10-11.

32 Breuer (1984), 35. 
deren von Autoren der Empfindsamkeit. ${ }^{33}$ Doch damit nicht genug: In den letzten Jahren ließ sich eine eigentliche Wiederentdeckung der Wüstenväter-Literatur beobachten. Psychiater und Psychotherapeuten nutzten sie, nun allerdings weder als Grundtexte christlicher Spiritualität noch als Zeugnisse asketischen Lebens, sondern als psychotherapeutisches Vademecum für einen erneuerten Umgang mit emotionalen und psychophysischen Problemen. ${ }^{34}$

\section{Negation}

Im literarischen Umfeld der spätmittelalterlichen Dominikanermystik wird die Wüste zu einem Metatopos. Sie veranschaulicht etwas, das sich vom Bildhaften eines Bildes so sehr unterscheidet, dass es zum Topos für die Bildlosigkeit an sich wird. Mit diesem Schritt in die Selbstreflexivität des Bildes wird die >Leiter ২ der wie auch immer gearteten geografischen, rhetorischen oder hermeneutischen Referenz, ja der Referentialität überhaupt, weggestoßen. Genau dazu fordert das Lied Granum sinapis (〉Senfkorn ‘) auf.

Das mystische Gedicht stammt aus Meister Eckharts Umkreis und lässt sich überlieferungsgeschichtlich ins frühe 14. Jahrhundert in Thüringen datieren. Seine Strophenform und die ebenfalls erhaltene Melodie weisen es als sangbar aus. ${ }^{35}$ Der Titel selbst entstammt dem mitüberlieferten scholastischen Kommentar und bezieht sich auf das Lied selbst. Das poetische Senfkorn bestehe nur aus wenigen Wörtern und sei doch groß an Wirkung (parvum in substantia, magnum in virtute), denn der Stoff dieses Büchleins (materia libelli) entfalte seine »überhimmlischen « Wirkungen, wie der Kommentar mit einer medizinischen Analogie zur pharmakologischen Anwendung des Senfkorns erklärt. Eine davon ist, dass der Geist über sich selbst hinausgeht (facit hoc excedere mentis) und im Blick auf die appellhaften beiden letzten Strophen ist es tatsächlich die wichtigste. ${ }^{36}$

Die ersten drei Strophen preisen die göttliche Trinität und die neuplatonisch gedachte Emanation des Wortes, dort, »dâ ie begin begin gebâr« (da stets Beginn Beginn gebar, 1,5) und alles Seiende ausfloss. Die vierte Strophe, die von den Wegen der Gotteserkenntnis spricht, führt das Bild der Wüste für die Gottheit ein und stellt mit deren Wesensbestimmung zugleich die Bedingung für die Erkennt-

$33 \mathrm{Zu}$ den Frühdrucken mit Wüstenväter-Texten und zu deren Rezeption bis ins 18. Jahrhundert s. Williams/Hoffmann (1999).

34 Vgl. Sartory (1992) und unter den zahlreichen, den Wüstenvätern gewidmeten Publikationen von Daniel Hell, Ordinarius für Psychiatrie an der Universität Zürich und Direktor der psychiatrischen Universitätsklinik, s. Hell (2005a); Hell (2005b).

35 Ruh (1981), 220-224. Mittelhochdeutscher Text und lateinischer Kommentar in Bindschedler (1949), neuhochdeutsche Übersetzung in Wehrli (1988), 486-493. Eine Audio-Fassung (mit zweisprachiger Textwiedergabe im Booklet) in Hamburger/Keller (2005). Zum Granum sinapis siehe Ruh (1989), 47-59; Haug (2003). - Die Interpretation folgt der in Keller (2008a) vorgelegten Fassung.

36 Bindschedler (1949), Komm. 2, 1, 34-35. 
nis fest: Da die Wüste zunächst unwegsam, breit, weit und unermesslich, dann, mit einem Paradoxon, auch ein Topos genannt wird, der weder zît noch stât (»Zeit noch Ort«) habe und deshalb sowohl »wunderbar« als auch »von ganz eigenem Dasein« genannt wird, ist der Weg dorthin ein Nicht-Weg. Noch nie, so die fünfte Strophe, habe ihn ein Fuß beschritten, noch nie ein Mensch mit den ihm zur Verfügung stehenden, begrifflichen Mitteln berührt, denn von ihm lässt sich nichts Distinktives benennen: kein Hier oder Dort, kein Fern oder Nah, kein Dies oder Das.

Das wûste gût

nî vûz durch wût, geschaffen $\sin$ quam nî dâ hin:

us ist und weis doch nimant was.

us hî, us dâ,

us verre, us nâ,

us tîf, us hô,

us ist alsô,

daz us ist weder diz noch daz.

(Strophe V)
Die Wüste, dieses Gut

durchschritt nie ein Fuß,

geschaffener Sinn

gelangte nie da hin:

es ist, und niemand weiß, was es ist.

es ist hier, es ist da,

es ist ferne, es ist nah,

es ist tief, es ist hoch,

es ist so beschaffen,

dass es weder dies noch das ist.

Die sechste Strophe vertieft die im Bild der Wüste liegende Paradoxie, dass sie ein Bild für das in sich selbst Bildlose sowie für das sich auch allen externen, wie auch immer beschaffenen diskursiven Abbildungen Entzogene ist: unbenant, unbekant (VI, 2-3).

Entgegen allen Warnungen, dass aus dem Wüstenbild keine Handlungsanweisung herzuleiten sei, empfiehlt die siebte Strophe Wege in die Wüste. Sie zu gehen bedeutete nichts anderes als eine erkenntnistheoretische Prämisse zu erfüllen: die Angleichung dessen, der erkennen soll (〉Subjektく kann er nicht mehr genannt werden), an das zu Erkennende. Der Weg in die Wüste besteht aus Negationen:

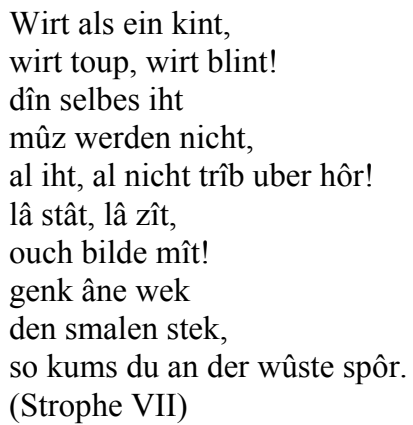

Werde wie ein Kind, werde taub, werde blind!

Dein eigenes Ich muß zunichte werden, alles Etwas und alles Nichts treibe hinweg! Laß Raum, laß Zeit, meide auch das Bild! Gehe ohne Weg den schmalen Pfad, dann findest du der Wüste Fußspur.

Und doch steigert sich die Dynamik zum Ende hin. In der letzten Strophe reißt sich das sprechende Ich mit mehrmaligen Selbstaufrufen aus sich selbst heraus, um sich in den (erst hier genannten) deus absconditus (der verborgene Gott) hineinzuwerfen, um in seine Flut zu sinken. Wundersam - und das ist zugleich das Wundersame am Wüsten-Topos - ist der hier in einzigartiger Klarheit zu beo- 
de Foucauld, Charles: Writing Selected, eingel. v. Robert Ellsberg, Maryknoll NY 1999 (= Modern Spiritual Masters).

Deutsche Lyrik des Mittelalters. Mit 36 Abbildungen aus der Manessischen Liederhandschrift, hg. v. Max Wehrli, 7. Aufl. Zürich 1988 (= Manesse Bibliothek der Weltliteratur).

Evagrios Pontikos: Briefe aus der Wüste, eingel., übs. u. komm. v. Gabriel Bunge, Trier 1986 (= Sophia, 24).

Seuse, Heinrich: Deutsche Schriften, im Auftrag der Württembergischen Kommission für Landesgeschichte hg v. Karl Bihlmeyer, Frankfurt/M. 1961 [Nachdruck der Ausgabe Stuttgart 1907].

Stimmen aus mittelalterlichen Frauenklöstern. Ein Hörbuch mit geistlichen Texten auf Altsächsisch, Mittelhochdeutsch und Mittelniederdeutsch, hg. v. Jeffrey F. Hamburger/Hildegard Elisabeth Keller, Berlin 2005.

Vie d'Antoine, hg. v. Gérard J. M. Bartelink, Paris 1994 (= Sources chrétiennes, 400).

Weisung der Väter. Apophthegmata Patrum, auch Gerontikon oder Alphabeticum genannt, hg. v. Bonifaz Miller, 5. Aufl. Trier 2002 (=Sophia, 6).

\section{Forschungsliteratur}

Asmuth, Christoph, ")... sô wonete der mensche in der wüestunge ...<. Meister Eckharts philosophischer Begriff der Wüste«, in: Was ist eine Wüste? Interdisziplinäre Annäherungen an einen interkulturellen Topos, hg. v. Uwe Lindemann/Monika Schmitz-Emans, Würzburg 2000, 115-126 (= Saarbrücker Beiträge zur vergleichenden Literatur- und Kulturwissenschaft, 12).

Bindschedler, Maria, Der lateinische Kommentar zum Granum sinapis, Basel 1949 (= Basler Studien zur deutschen Sprache und Literatur, 9).

Bost, Hubert, »Le désert des Huguenots: une poétique de l'épreuve«, in: Le Désert: L'espace et l'esprit (Moyen Age - XX $\mathrm{X}^{\mathrm{e}}$ siècle), hg. v. Frank Lestringant/Sarga Moussa, Lille 2000 (= Revue des Sciences Humaines, 258), 177206.

Breuer, Dieter, "Zur Druckgeschichte und Rezeption der Schriften Heinrich Seuses«, in: Frömmigkeit in der frühen Neuzeit. Studien zur religiösen Literatur des 17. Jahrhunderts in Deutschland, hg. v. Dieter Breuer, Amsterdam 1984 (= Chloe. Beihefte zum Daphnis, 2), 29-49.

Brown, Peter, Die Keuschheit der Engel. Sexuelle Entsagung, Askese und Körperlichkeit im frühen Christentum, übs. v. Martin Pfeiffer, München 1994.

Brunert, Maria-Elisabeth, »Die Bedeutung der Wüste im Eremitentum«, in: Was ist eine Wüste? Interdisziplinäre Annäherungen an einen interkulturellen Topos, hg. v. Uwe Lindemann/Monika Schmitz-Emans, Würzburg 2000 
(= Saarbrücker Beiträge zur vergleichenden Literatur- und Kulturwissenschaft, 12), 59-69.

Burden, Terry L., The Kerygma of the Wilderness Tradition in the Hebrew Bible, New York 1994 (= American University Studies. Series VII: Theology and Religion, 163).

Dietrich, Paul A., »The Wilderness of God in Hadewijch II and Meister Eckhart and His Circle«, in: Meister Eckhart and the Beguine Mystics. Hadewijch of Brabant, Mechthild of Magdeburg, and Marguerite Porete, hg. v. Bernard McGinn, New York 1994.

Donner, Herbert, » Die religiöse Wüstenromantik. Über eine fehlgeleitete Metapher«, in: Arbeiten zur Praktischen Theologie, hg. v. Volker et al. Drehsen, Bd. 10. Berlin/New York 1997, 3-12.

Fragonard, Marie-Madeleine, »Désert romanesques et dévotion aisée«, in: Le Désert: L'espace et l'esprit (Moyen Age - XX siècle), hg. v. Frank Lestringant/Sarga Moussa, Lille 2000 (= Revue des Sciences Humaines, 258), 163176.

Gnädinger, Louise, »Arsenius. Ein bevorzugter geistlicher Lehrmeister Heinrich Seuses«, in: Heinrich Seuse - Diener der Ewigen Weisheit, hg. v. Jakobus Kaffanke, Freiburg i. Br. 1998 (= Tagungsberichte der Katholischen Akademie der Erzdiözese Freiburg), 87-159.

Goehring, James E., »The Encroaching Desert: Literary Production and Ascetic Space in Early Christian Egypt«, in: Journal of Early Christian Studies 1, 3 (1993), 281-296.

Goehring, James E., Ascetics, Society, and the Desert: Studies in Early Egyptian Monasticism, Harrisburg 1999 (= Studies in Antiquity and Christianity).

Goehring, James E., »The Dark Side of Landscape: Ideology and Power in the Christian Myth of the Desert«, in: Journal of Medieval and Early Modern Studies 33, 3 (2003), 437-451.

Gomes de Araujo, Reginaldo, Theologie der Wüste im Deuteronium, Frankfurt/M. 1999 (= Österreichische Biblische Studien, 17).

Hamburger, Jeffrey F., »Die Orte«, in: Die Stunde des Hundes. Auf dem mystischen Weg zu Gott. Ein Hörbuch nach Heinrich Seuses Exemplar, hg. v. Hildegard Elisabeth Keller, Zürich 2007, 45-55.

Haug, Walter, »Meister Eckhart und das \Granum sinapis««; in: Forschungen zur deutschen Literatur des Spätmittelalters. Festschrift für Johannes Janota, hg. v. Horst Brunner/Werner Williams-Krapp, Tübingen 2003, 73-92.

Hell (2005a), Daniel, Die Sprache der Seele verstehen. Die Wüstenväter als Therapeuten, 6. Aufl. Freiburg im Breisgau (= Herder spektrum, 5191).

Hell (2005b), Daniel, Leben als Geschenk und Antwort. Weisheiten der Wüstenväter, Freiburg i. Br. (= Herder spektrum, 5624).

Keller (2008a), Hildegard Elisabeth, »Wüste. Porträt eines Nicht-Ortes«, in: Burgen, Länder, Orte, hg. v. Werner Wunderlich/Ulrich Müller, St. Gallen 2008, 926-941 (= Mittelalter-Mythen, 5), 997-1007. 
Keller (2008b), Hildegard Elisabeth, »Wald, Wälder. Streifzüge durch einen Topos«, in: Burgen, Länder, Orte, hg. v. Werner Wunderlich/Ulrich Müller, St. Gallen 2008, 926-941 (= Mittelalter-Mythen, 5).

Keller, Hildegard Elisabeth, Die Stunde des Hundes. Auf dem mystischen Weg zu Gott. Ein Hörbuch nach Heinrich Seuses Exemplar, Zürich 2007.

Kunze, Konrad/Ulla Williams/Philipp Kaiser, »Information und innere Formung. Zur Rezeption der >Vitaspatrum««, in: Wissensorganisierende und wissensvermittelnde Literatur im Mittelalter, hg. v. Norbert Richard Wolf, Wiesbaden 1987 (= Wissensliteratur im Mittelalter, 1), 123-142.

Le Désert: L'espace et l'esprit (Moyen Age - XX siècle), hg. v. Frank Lestringant/Sarga Moussa, Lille 2000 (= Revue des Sciences Humaines, 258).

Lindemann, Uwe: Die Wüste. Terra incognita - Erlebnis - Symbol. Eine Genealogie der abendländischen Wüstenvorstellungen in der Literatur von der Antike bis zur Gegenwart, Heidelberg 2000 (= Beiträge zur neueren Literaturgeschichte, 175).

Lipp, Wolfgang: »Selbststigmatisierung«, in: Stigmatisierung, Bd. 1: Zur Produktion gesellschaftlicher Randgruppen, hg. v. Manfred Brusten/Jürgen Hohmeier, Darmstadt 1975, 25-53.

Lipp, Wolfgang: Stigma und Charisma. Über soziales Grenzverhalten, Berlin 1985 (= Schriften zur Kultursoziologie, 1).

McGinn, Bernard: »Ocean and Desert as Symbols of Mystical Absorption in the Christian Tradition«, in: The Journal of Religion 74/2 (1994), 155-181.

Michel, Paul/Deeg, Stefan/Goldschmidt, Hermann Levin: »Symbolik der Wüste«, in: Symbolik von Ort und Raum, hg. v. Paul Michel, Bern 1997 (=Schriften der Schweizerischen Gesellschaft für Symbolforschung, 11), 159-216.

Müller, Barbara: Der Weg des Weinens. Die Tradition des >Penthos` in den Apophthegmata Patrum, Göttingen 2000 (=Forschungen zur Kirchen- und Dogmengeschichte, 77).

Mödritzer, Helmut: Stigma und Charisma im Neuen Testament und seiner Umwelt. Zur Soziologie des Urchristentums, Freiburg i. d. Schweiz/Göttingen 1994 (= Novum Testamentum et Orbis Antiquus, 28).

Petroff, Elizabeth Alvilda: ") She Seemed to Have Come from the Desert<: Italian Women Saints and the Vitae Patrum Cycle«, in: Body and Soul. Essays on Medieval Women and Mysticism, hg. v. Elizabeth Alvilda Petroff, New York/Oxford 1994, 110-138.

Rahner, Karl: Sämtliche Werke. Bd. 3: Spiritualität und Theologie der Kirchenväter, bearb. v. Andreas R. Batlogg/Eduard Farrugia/Karl-Heinz Neufeld, Freiburg i. Br. 1999.

Ruh, Kurt: Art. »Granum sinapis«, in: Die deutsche Literatur des Mittelalters. Verfasserlexikon, Bd. 3 (1981), Sp. 220-224.

Ruh, Kurt: Meister Eckhart. Theologe - Prediger - Mystiker, 2. Aufl. München 1989.

Sartory, Gertrude/Thomas Sartory: Lebenshilfe aus der Wüste. Die alten Mönchsväter als Therapeuten, 8. Aufl. Freiburg i. Br. 1992. 
Stolz, Fritz: Weltbilder der Religionen. Kultur und Natur, Diesseits und Jenseits, Kontrollierbares und Unkontrollierbares, Zürich 2001 (= Theophil. Zürcher Beiträge zu Religion und Philosophie, 4).

Williams, Ulla/Werner J. Hoffmann, Art. »Vitaspatrum«, in: Die deutsche Literatur des Mittelalters. Verfasserlexikon, Bd. 10 (1999), Sp. 449-466. 